\title{
Synthesis of the reported structure of piperazirum using a nitro-Mannich reaction as the key stereochemical determining step
}

\author{
James C. Anderson ${ }^{* 1}$, Andreas S. Kalogirou ${ }^{1}$, Michael J. Porter ${ }^{1}$
}

and Graham J. Tizzard ${ }^{2, \S}$

\section{Letter}

\section{Address:}

${ }^{1}$ Department of Chemistry, University College London, 20 Gordon Street, London, WC1H OAJ, UK and ${ }^{2}$ National Crystallography Service, School of Chemistry, University of Southampton,

Southampton, SO17 1BJ, UK

\section{Email:}

James C. Anderson* - j.c.anderson@ucl.ac.uk; Graham J. Tizzard gjt1@soton.ac.uk

* Corresponding author

$\S$ Corresponding author for crystallographic results

Keywords:

alkaloid; aza-Henry; natural products; nitro-Mannich; piperazinone; stereoselective synthesis
Beilstein J. Org. Chem. 2013, 9, 1737-1744.

doi:10.3762/bjoc. 9.200

Received: 10 July 2013

Accepted: 01 August 2013

Published: 23 August 2013

This article is part of the Thematic Series "Natural products in synthesis and biosynthesis".

Guest Editor: J. S. Dickschat

(C) 2013 Anderson et al; licensee Beilstein-Institut.

License and terms: see end of document.

\begin{abstract}
Piperazirum, isolated from Arum palaestinum Boiss, was originally assigned as $r$-3,c-5-diisobutyl-c-6-isopropylpiperazin-2-one. The reported structure was synthesised diastereoselectively using a key nitro-Mannich reaction to set up the C5/C6 relative stereochemistry. The structure was unambiguously assigned by single crystal X-ray diffraction but the spectroscopic data did not match those reported for the natural product. The structure of the natural product must therefore be revised.
\end{abstract}

\section{Introduction}

The nitro-Mannich reaction (or aza-Henry reaction) has been developed to a standard where the product $\beta$-nitroamines $\mathbf{1}$ are now privileged building blocks. In part this is due to the complementary synthetic flexibility available from the two different nitrogen atom oxidation states (Scheme 1) [1]. They have been used to synthesise many nitrogen-containing functional groups including $\alpha$-amino carbonyls $[2,3]$, peptidomimetics [4], natural products [5-10] and many heterocyclic small molecules [11-24] of importance to drug discovery. Enantioselective reactions have been controlled by asymmetric metalcentred Lewis acids; chiral hydrogen bond donors, in particular by the use of asymmetric thiourea organocatalysts, chiral Brønsted acids, phase-transfer catalysts and Brønsted base catalysts [3,15,25-39]. A large part of our own work in developing the nitro-Mannich reaction was to demonstrate the preparation of stereodefined 1,2-diamines [40-45]. 


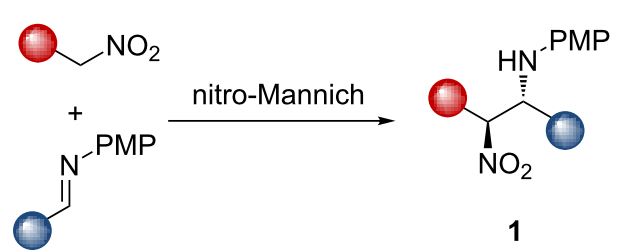

Scheme 1: Schematic nitro-Mannich reaction.

As part of a programme aimed at using these 1,2-diamines as building blocks in target synthesis we focused on the synthesis of a novel bioactive alkaloid, piperazirum (2, Scheme 2$)$, which to our knowledge had not been previously synthesised. Piperazirum was isolated from the leaf extract of Arum palaestinum Boiss and was shown to possess significant cytotoxicity against cultured tumor cell lines in vitro [46]. Its chemical structure and relative stereochemistry were elucidated by high resolution mass spectrometry, infrared, 1D and 2D NMR spectroscopy [46]. Retrosynthetically we envisaged that the C-3 stereocentre could be set up by hydrogenation from the less hindered face of $\alpha$-iminolactam 3 or $\alpha, \beta$-unsaturated lactam 4 (Scheme 2). These heterocycles could be derived from a common $\alpha$-keto acid derivative 5 and either diamine 6 or 7 , that could in turn be prepared from $\beta$-nitroamine $\mathbf{8}$ or $\mathbf{9}$. Each of the $\beta$-nitroamines could be prepared enantioselectively by using our previously reported methodology $[28,43,45]$ and would allow elucidation of the absolute stereochemistry of piperazirum (2).

\section{Results and Discussion}

The common $\alpha$-keto acid derivative $\mathbf{5}$ was easily prepared from a Grignard reaction of isobutylmagnesium chloride with diethyl oxalate to give $\alpha$-keto ester 10 in 94\% yield (Scheme 3) [47]. Saponification of $\mathbf{1 0}$ with $\mathrm{KOH}$ provided $\alpha$-keto acid $\mathbf{1 1}$ in excellent yield [48], and the corresponding acid chloride 12 was prepared in situ by treatment with oxalyl chloride [49].

For the synthesis of $\beta$-nitroamine $\mathbf{8}$ we decided to make use of the reductive nitro-Mannich reaction as the starting nitroalkene

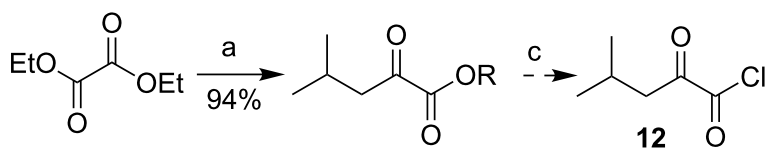

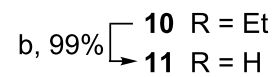

Scheme 3: (a) iBuMgCl, $\mathrm{Et}_{2} \mathrm{O},-78{ }^{\circ} \mathrm{C}$; (b) $\mathrm{KOH}, \mathrm{EtOH} / \mathrm{H}_{2} \mathrm{O}, 100{ }^{\circ} \mathrm{C}$; (c) $(\mathrm{COCl})_{2}$.

$\mathbf{1 3}$ is readily available from a Henry reaction [50], imine $\mathbf{1 4}$ from the condensation of $p$-anisidine and isobutyraldehyde, and the process can easily be made asymmetric [45]. Conjugate addition of hydride to $\mathbf{1 3}$ and subsequent trapping of the nitronate anion with freshly prepared imine $\mathbf{1 4}$ in THF gave $\beta$-nitroamine 15 in 64\% conversion and a dr of 70:30 (Scheme 4). Quite frequently $\beta$-nitroamines are unstable and susceptible to retroaddition $[43,44]$. Formation of the corresponding trifluoroacetamide derivative confers stability and allows them to be purified. Using previously developed conditions to protect the amine in situ using $\left(\mathrm{CF}_{3} \mathrm{CO}\right)_{2} \mathrm{O}$ gave only $\beta$-nitroamine $\mathbf{1 6}$ as a single diastereoisomer in a low $15 \%$ yield. These results are consistent with the poor conversions and dr, as well as resistance to trifluoroacetamide protection, we have observed before from imines derived from $\alpha$-branched aldehydes such as cyclohexanecarbaldehyde [44].

In cases where trifluoroacetylation fails, it is quite common to purify the $\beta$-nitroamine by rapid column chromatography, quickly followed by reduction to give the corresponding 1,2-diamine. In this case rapid purification of $\mathbf{1 5}$ followed by reduction with $\mathrm{Zn} / \mathrm{HCl}$ gave the 1,2-diamine $\mathbf{1 7}$ as a single diastereoisomer in $50 \%$ yield (Scheme 4 ).

With diamine $\mathbf{1 7}$ in hand, the reaction with a suitable keto acid derivative was investigated. We presumed that the nitrogen of the primary amine would be the more nucleophilic and hence should react with the more electrophilic carbonyl group - i.e.

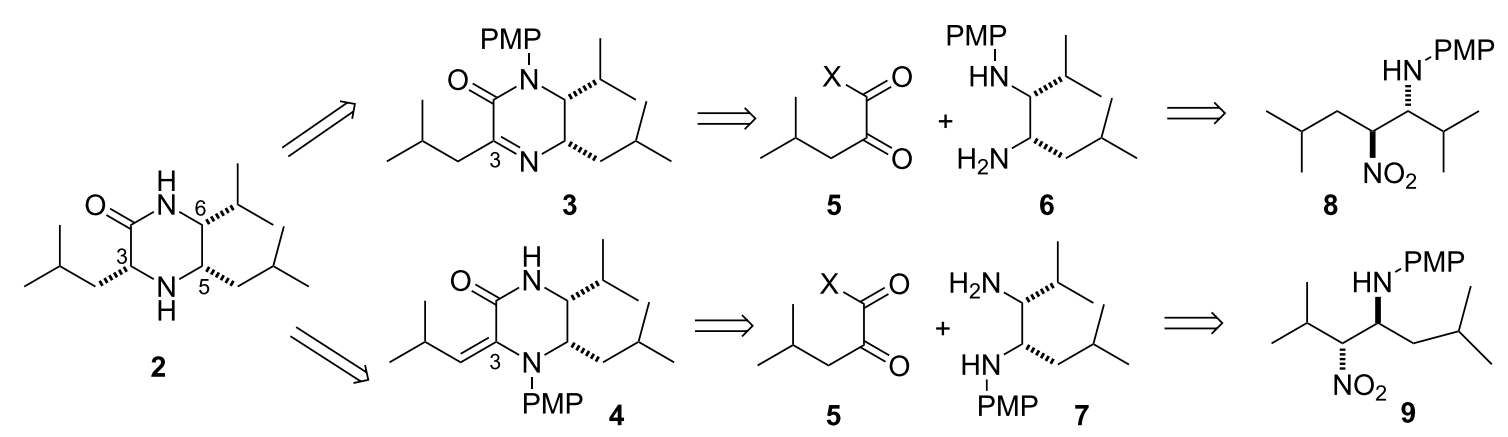




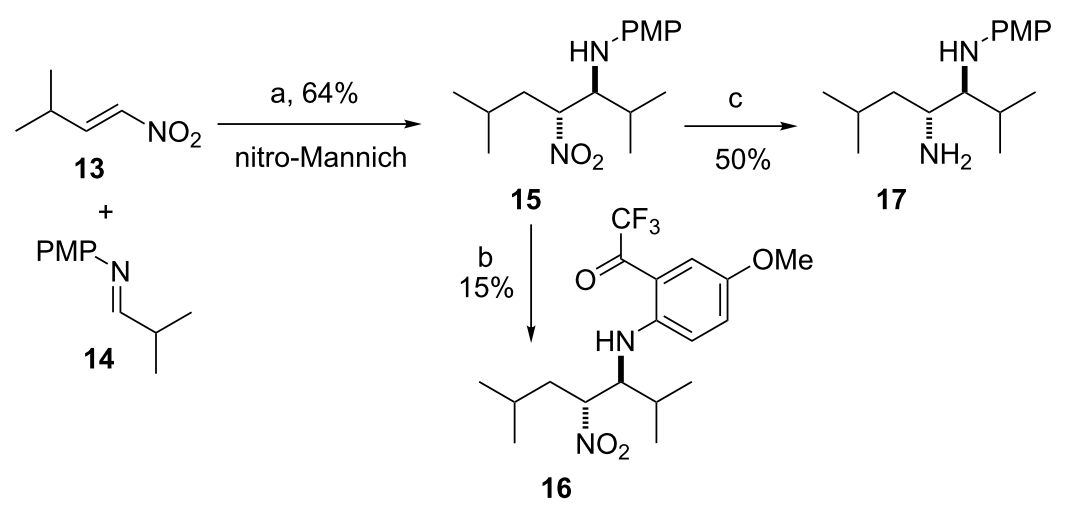

Scheme 4: (a) $\mathrm{Li}\left(\mathrm{Et}_{3} \mathrm{BH}\right)$, THF, rt then 14, $\mathrm{CF}_{3} \mathrm{CO}_{2} \mathrm{H},-78^{\circ} \mathrm{C}$, dr 70:30; (b) $\left(\mathrm{CF}_{3} \mathrm{CO}\right)_{2} \mathrm{O}, \mathrm{Py}, \mathrm{CH}_{2} \mathrm{Cl}_{2}, 0^{\circ} \mathrm{C}$ to rt; (c) $\mathrm{Zn}, 6 \mathrm{M} \mathrm{HCl}, \mathrm{EtOAc} / \mathrm{EtOH}, \mathrm{rt}$.

the ketone - in $\mathbf{1 0}$ [51,52]. Attempted formation of $\mathbf{4}$ by heating a mixture of $\mathbf{1 0}$ and $\mathbf{1 7}$ in $\mathrm{H}_{2} \mathrm{O}$ at $50{ }^{\circ} \mathrm{C}$ gave a complicated mixture of products and attempts under Dean-Stark conditions in toluene with $\mathrm{TsOH}$ gave only recovered starting materials [53].

In light of this poor result, the alternative route to $\mathbf{2}$ via $\beta$-nitroamine 9 was investigated. A reductive nitro-Mannich reaction between nitroalkene $\mathbf{1 8}$ [54] and freshly prepared imine 19 in $\mathrm{CH}_{2} \mathrm{Cl}_{2}$ followed by rapid flash chromatography gave $\beta$-nitroamine 20 with complete conversion and $\mathrm{dr}>95: 5$ [55]. As before immediate reduction with $\mathrm{Zn} / \mathrm{HCl}$ gave the PMPprotected diamine $\mathbf{2 1}$ in $85 \%$ yield as a single diastereoisomer determined by ${ }^{1} \mathrm{H}$ NMR (Scheme 5).

From a comprehensive series of examples of the reductive nitro-Mannich reaction, the vast majority of substrates demonstrate anti-relative stereochemistry [23,44,45]. More direct proof for $\mathbf{2 1}$ was gleaned from the corresponding imidazolidine2-thione formed by treatment with thiophosgene to give $\mathbf{2 2}$ (Scheme 5). In one dimensional nOe studies irradiation of the $\mathrm{C} H \mathrm{NH}$ peak $(\delta 3.70,1 \mathrm{H}, \mathrm{dd}, J=8.4,5.4 \mathrm{~Hz})$ caused a $3.65 \%$ enhancement of the $\mathrm{C} H \mathrm{~N}$ peak $(\delta 4.31,1 \mathrm{H}, \mathrm{dt}, J=7.9,5.9 \mathrm{~Hz})$, indicating a cis-relative stereochemistry between the two protons, which confirmed the anti-relative stereochemistry of
21. The observed coupling constant between the same two protons was $8.2 \mathrm{~Hz}$ (averaged) and was similar to other imidazolidine-2-thiones we have prepared that have been corroborated by single crystal X-ray crystallography [56]. Further stereochemical proof was provided by a single crystal $\mathrm{X}$-ray structure determination of $\mathbf{2} \cdot \mathrm{HCl}$ (vide infra).

We presumed again that the primary amine of $\mathbf{2 1}$ would be more nucleophilic towards a keto acid derivative $\mathbf{5}$. In order to obtain a piperazinone of the desired connectivity (23) a keto acid derivative would be required where the carboxylate carbonyl is more reactive than the ketone carbonyl. Two possible such compounds were considered, acid chloride $\mathbf{1 2}$ and carboxylic acid $\mathbf{1 1}$ treated with a suitable coupling agent. Acid chloride $\mathbf{1 2}$ was prepared in situ by treatment of acid $\mathbf{1 1}$ with oxalyl chloride (2.00 equiv) and catalytic DMF. Subsequent reaction with diamine $\mathbf{2 1}$ in the presence of pyridine (1.20 equiv) and catalytic DMAP over $24 \mathrm{~h}$, according to previously reported reactions for similar keto acids [49], gave only the bis-adduct $\mathbf{2 4}$ and none of the desired piperazinone 23. By contrast, the reaction of carboxylic acid 11 with diamine 21, in the presence of EDC (1.50 equiv) and 1-hydroxybenzotriazole ( 1.50 equiv) at $\mathrm{rt}$, gave the desired product $\mathbf{2 3}$ in good yield (Scheme 6) [57]. The double bond geometry was assigned as $Z$ by NOESY ${ }^{1} \mathrm{H}$ NMR and probably results from steric inhibi-

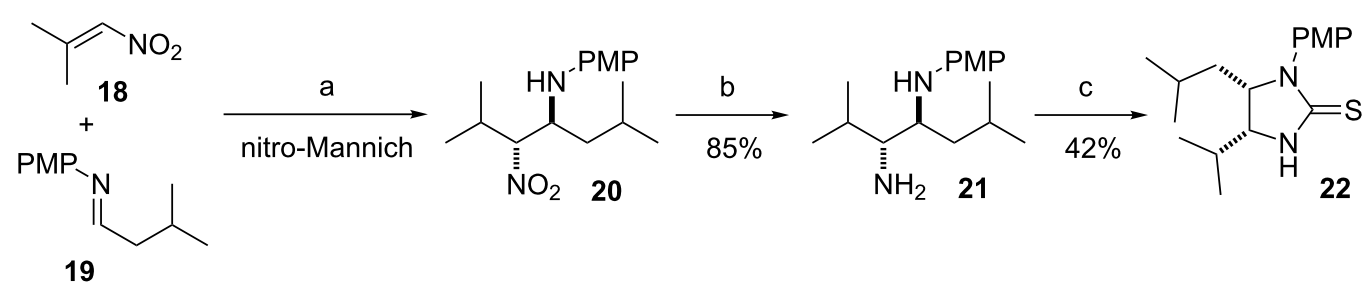

Scheme 5: (a) $\mathrm{Li}\left(\mathrm{Et}_{3} \mathrm{BH}\right), \mathrm{CH}_{2} \mathrm{Cl}_{2}$, rt then 19, $\mathrm{CF}_{3} \mathrm{CO}_{2} \mathrm{H},-78{ }^{\circ} \mathrm{C}, \mathrm{dr}>95: 5$; (b) $\mathrm{Zn}, 6 \mathrm{M} \mathrm{HCl}, \mathrm{EtOAc} / \mathrm{EtOH}$, rt, single diastereoisomer; (c) $\mathrm{CSCl}_{2}$, aq $\mathrm{NaHCO}_{3} \mathrm{CH}_{2} \mathrm{Cl}_{2} / \mathrm{MeOH}$, rt. 


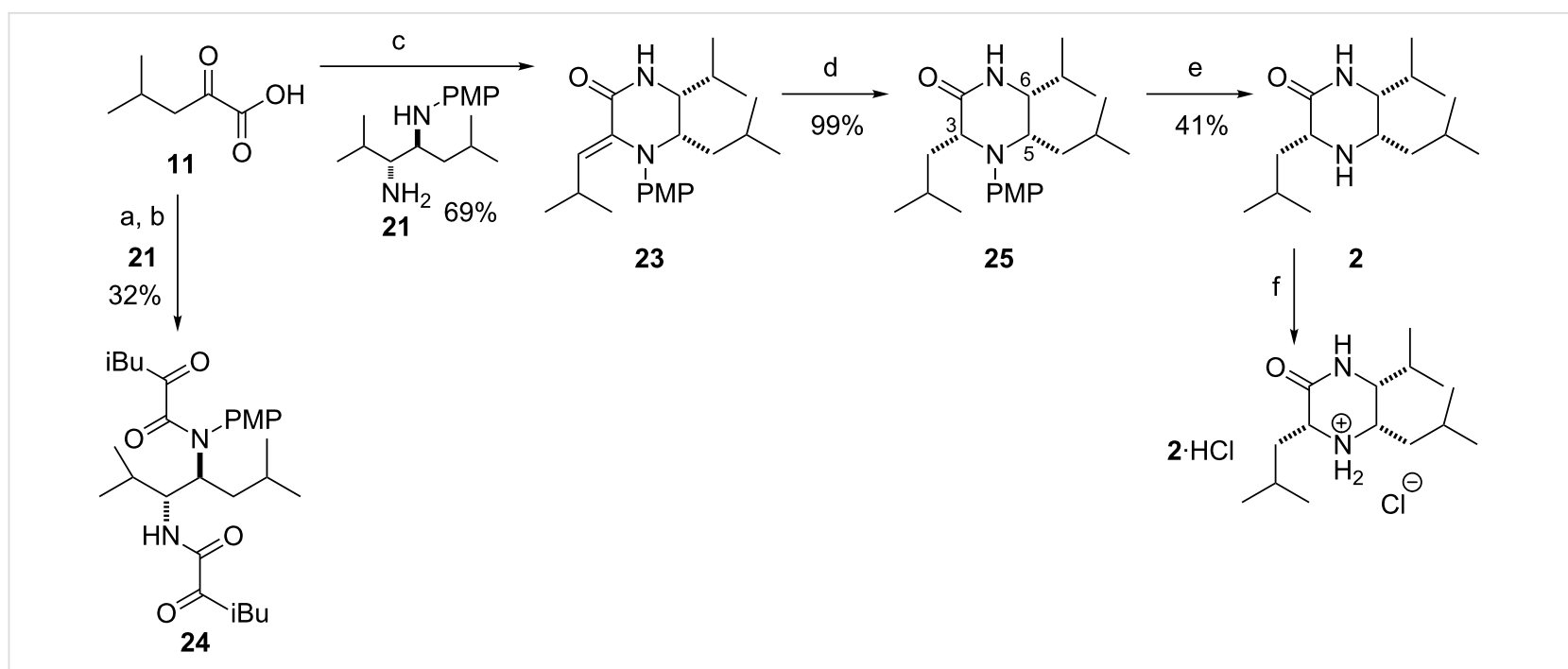

Scheme 6: (a) $(\mathrm{COCl})_{2}, \mathrm{DMF}, \mathrm{CH}_{2} \mathrm{Cl}_{2}$, rt; (b) 21, Py, DMAP, $\mathrm{CH}_{2} \mathrm{Cl}_{2}$, rt; (c) EDC, $\mathrm{HOBt}, \mathrm{THF} / \mathrm{CH}_{2} \mathrm{Cl}_{2}, \mathrm{rt}$; (d) $\mathrm{H}_{2}(1 \mathrm{~atm}), \mathrm{Pd} / \mathrm{C}, \mathrm{MeOH}, \mathrm{rt}$; (e) CAN, $\mathrm{MeCN} / \mathrm{H}_{2} \mathrm{O}, 0^{\circ} \mathrm{C}$; (f) $4 \mathrm{M} \mathrm{HCl}$ in dioxane, $\mathrm{MeCN} / \mathrm{Et}_{2} \mathrm{O}$.

tion of resonance which would result in the formation of the $E$ iPr group and the planar amide group during formation of 23 .

Reduction of the double bond with hydrogen over palladium on charcoal gave a single diastereoisomer $\mathbf{2 5}$ in quantitative yield. In one dimensional nOe studies irradiation of $\mathrm{H}-3(\delta 4.09,1 \mathrm{H}$, dd, $J=9.9,4.5 \mathrm{~Hz}$ ) caused a $0.17 \%$ enhancement of the H- 6 signal $(\delta 3.18,1 \mathrm{H}, \mathrm{dd}, J=10.0,3.5 \mathrm{~Hz})$ with negligible enhancement of the H-5 signal $(\delta 3.37,1 \mathrm{H}, \mathrm{dt}, J=12.4,3.1$ $\mathrm{Hz}$ ). Irradiation of H-5 caused a $2.90 \%$ enhancement of the H- 6 signal and a $0.07 \%$ enhancement of the $\mathrm{H}-3$ signal. The nOe data tentatively suggested that protons H-3, H-5 and H-6 were all on the same face of the molecule. Deprotection of the PMP group with CAN gave compound 2. Extensive NMR (COSY, HMBC and NOESY) and analytical data were consistent with the structure drawn (2), but the ${ }^{1} \mathrm{H}$ and ${ }^{13} \mathrm{C}$ NMR did not match that published for piperazirum (Table 1) [46]. The original authors recorded their NMR data for piperazirum in $\mathrm{D}_{2} \mathrm{O}$ [58], but our sample 2 was insoluble. Compound 2 was readily soluble in DMSO- $d_{6}$ and $\mathrm{CDCl}_{3}$, but neither gave a satisfactory match to the reported NMR data. Preparation of $2 \cdot \mathrm{HCl}$ allowed the recording of NMR spectra in $\mathrm{D}_{2} \mathrm{O}$, but again the chemical shifts were inconsistent with those reported. Single crystal $\mathrm{X}$-ray structure determination of $\mathbf{2}$ proved unambiguously the assigned structure obtained from spectroscopic data (Figure 1) [59].

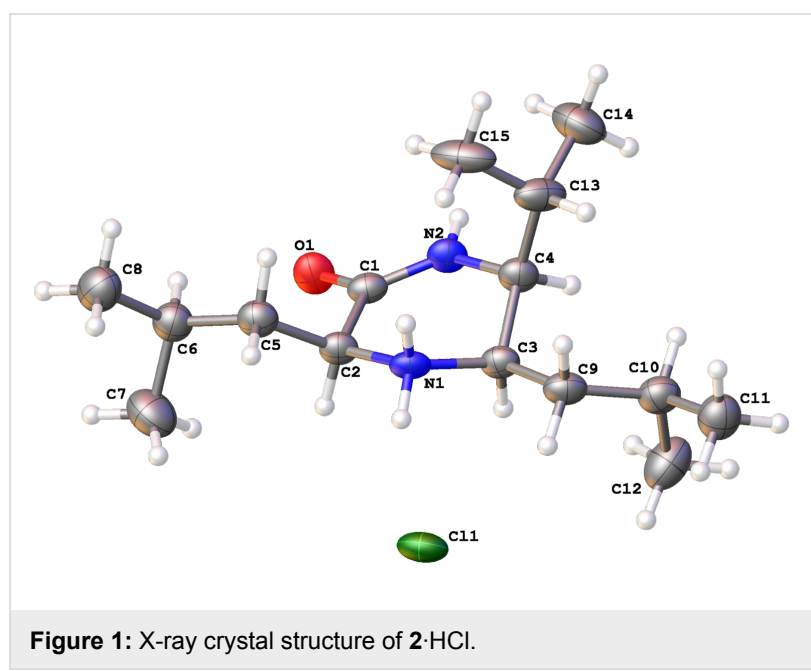

Table 1: Comparison of selected ${ }^{13} \mathrm{C}$ NMR chemical shifts of piperazirum, 2 and $\mathbf{2} \cdot \mathrm{HCl}$.

\begin{tabular}{|c|c|c|c|c|}
\hline $\begin{array}{l}\text { Carbon } \\
\text { atom }\end{array}$ & $\begin{array}{l}\text { piperazirum } \delta c(p p m) \\
\qquad D_{2} \mathrm{O}[49]\end{array}$ & $\begin{array}{l}2 \delta c(p p m) \\
\text { DMSO-d }\end{array}$ & $\begin{array}{c}2 \delta c(p p m) \\
\mathrm{CDCl}_{3}\end{array}$ & $\begin{array}{c}2 \cdot \mathrm{HCl} \delta \mathrm{c}(\mathrm{ppm}) \\
\mathrm{D}_{2} \mathrm{O}\end{array}$ \\
\hline $\mathrm{C}-2$ & 175.7 & 172.5 & 174.3 & 169.9 \\
\hline C-3 & 53.6 & 56.4 & 56.9 & 54.9 \\
\hline$C-5$ & 59.7 & 53.2 & 53.3 & 53.9 \\
\hline C-6 & 60.6 & 58.1 & 59.4 & 56.3 \\
\hline $\mathrm{CH}_{2} \mathrm{C}-3$ & 40.0 & 41.5 & 41.1 & 38.8 \\
\hline $\mathrm{CH}_{2} \mathrm{C}-5$ & 24.6 & 40.2 & 40.5 & 36.3 \\
\hline
\end{tabular}




\section{Conclusion}

The rapid synthesis of $r-3, c-5$-diisobutyl-c-6-isopropylpiperazin-2-one has been achieved using an efficient nitroMannich reaction to establish the C-5/C-6 relative stereochemistry which in turn controls the formation of the stereocentre at C-3. Spectrosocpic and single crystal X-ray data have shown that the reported structure for piperazirum is erroneous and that the structure of the natural product needs to be revised. While the reported data point strongly towards piperazirum having the same connectivity as $\mathbf{2}$, it is not clear which of the three alternative diastereomers corresponds to the natural product. In view of the lack of natural material, further chemical synthesis, guided by GIAO chemical shift prediction, is currently underway in an effort to elucidate the correct structure for piperazirum. In addition the determination of further biological activity of $\mathbf{2}$ and its diastereoisomers will be investigated.

\section{Experimental}

Unless otherwise stated, all reactions were carried out under an atmosphere of nitrogen. All glassware was flame dried and allowed to cool under a stream of nitrogen before use. Cooling to $0{ }^{\circ} \mathrm{C}$ was effected using an ice-water bath. Cooling to temperatures below $0{ }^{\circ} \mathrm{C}$ was effected using dry ice-acetone mixtures. Reactions were monitored by thin layer chromatography (TLC) using $0.25 \mathrm{~mm}$ silica precoated plastic plates with fluorescent indicator. Sheets were visualised using ultraviolet light $(254 \mathrm{~nm})$ and/or anisaldehyde or $\mathrm{KMnO}_{4}$ solutions, as appropriate. Removal of solvents (in vacuo) was achieved using a water aspirator and rotary evaporators. Flash column chromatography was performed using silica gel 60, 40-63 $\mu \mathrm{m}$. Commercial solvents and reagents were used as supplied or purified in accordance with standard procedures [60]. Diethyl ether $\left(\mathrm{Et}_{2} \mathrm{O}\right)$, tetrahydrofuran (THF), dichloromethane $\left(\mathrm{CH}_{2} \mathrm{Cl}_{2}\right)$ and toluene $(\mathrm{PhMe})$ were obtained from a solvent tower, where degassed solvent was passed through two columns of activated alumina and a 7 micron filter under 4 bar pressure. All NMR samples were made as dilute solutions of $\mathrm{CDCl}_{3}$ unless otherwise stated. All chemical shifts $(\delta)$ are reported in parts per million (ppm) relative to residual solvent peaks except in $\mathrm{D}_{2} \mathrm{O}$ where they are relative to dioxane $\left(\mathrm{D}_{2} \mathrm{O}\right){ }^{1} \mathrm{H} 3.75 \mathrm{ppm}$ and ${ }^{13} \mathrm{C}$ $67.2 \mathrm{ppm}$. Multiplicities for ${ }^{1} \mathrm{H}$ coupled signals are denoted as $\mathrm{s}=$ singlet, $\mathrm{d}=$ doublet, $\mathrm{t}=$ triplet, $\mathrm{q}=$ quartet, quint = quintet, $\mathrm{m}=$ multiplet. Coupling constants $(J)$ are reported in Hertz. ${ }^{13} \mathrm{C}$ multiplicities were assigned using a DEPT sequence. Where appropriate, HMQC, COSY, HMBC and NOE experiments were carried out to aid assignment. Melting points are uncorrected.

(E)-4-Methoxy- $\boldsymbol{N}$-(3-methylbutylidene)aniline (19): To a mixture of $p$-anisidine $(123 \mathrm{mg}, 1.00 \mathrm{mmol})$ and basic alumina $(1.00 \mathrm{~g})$ in $\mathrm{CH}_{2} \mathrm{Cl}_{2}(5 \mathrm{~mL})$ at $-78{ }^{\circ} \mathrm{C}$ was added isovaleralde- hyde $(107 \mu \mathrm{L}, 1.00 \mathrm{mmol})$ and the mixture stirred for $1 \mathrm{~h}$, then warmed to rt, filtered and evaporated in vacuo to give crude imine 19 (182 $\mathrm{mg}, 95 \%)$ as a colourless oil that was used immediately without further purification; ${ }^{1} \mathrm{H}$ NMR $(600 \mathrm{MHz}) \delta 1.02$ (d, $J=6.7,6 \mathrm{H}, \mathrm{CH}_{3}$ ), 2.04 (sept, $\left.J=6.7,1 \mathrm{H}, \mathrm{CH}\left(\mathrm{CH}_{3}\right)_{2}\right), 2.34$ (dd, $J=7.0,5.4,2 \mathrm{H}, \mathrm{CH}_{2}$ ), 3.80 (s, $1 \mathrm{H}, \mathrm{OCH}_{3}$ ), 6.87 (app. d, $J$ $=8.9,2 \mathrm{H}, \operatorname{Ar} H), 7.02($ app. $\mathrm{d}, J=8.9,2 \mathrm{H}, \operatorname{Ar} H), 7.86(\mathrm{t}, J=$ $5.4,1 \mathrm{H}, \mathrm{N}=\mathrm{CH}) ;{ }^{13} \mathrm{C}$ NMR $(150 \mathrm{MHz})$ data were in agreement to that reported [61].

$\left(3 R^{*}, 4 S^{*}\right)-N^{4}$-(4-Methoxyphenyl)-2,6-dimethylheptane-3,4diamine (21): To a solution of nitroalkene 18 (202 $\mathrm{mg}$, $2.00 \mathrm{mmol})$, in $\mathrm{CH}_{2} \mathrm{Cl}_{2}(12.0 \mathrm{~mL})$ was added Superhydride ${ }^{\circledR}$ (2.20 mL, $1 \mathrm{M}$ in THF, $2.20 \mathrm{mmol}$ ) and the mixture stirred for $15 \mathrm{~min}$ at $\mathrm{rt}$. The mixture was cooled to $-78^{\circ} \mathrm{C}$ before the dropwise addition of a solution of freshly prepared imine 19 (564 mg, $4.00 \mathrm{mmol}$ ) in $\mathrm{CH}_{2} \mathrm{Cl}_{2}(12.0 \mathrm{~mL}$ ). The reaction was stirred for $10 \mathrm{~min}$ before the dropwise addition of a solution of $\mathrm{CF}_{3} \mathrm{CO}_{2} \mathrm{H}(460 \mu \mathrm{L}, 6.00 \mathrm{mmol})$ in $\mathrm{CH}_{2} \mathrm{Cl}_{2}(4.0 \mathrm{~mL})$. The reaction was stirred for $1 \mathrm{~h}$ and then quenched with brine $(20 \mathrm{~mL})$ at $-78^{\circ} \mathrm{C}$, warmed to $\mathrm{rt}$ and extracted with $\mathrm{Et}_{2} \mathrm{O}(3 \times 20 \mathrm{~mL})$. The combined organics were dried $\left(\mathrm{MgSO}_{4}\right)$ and evaporated in vacuo to give crude $\beta$-nitroamine $\mathbf{2 0}$, that was purified by column chromatography (petrol ether/Et $\mathrm{t}_{2} \mathrm{O} 4: 1$ ). Subsequent reaction with $6 \mathrm{M} \mathrm{HCl}(6.60 \mathrm{~mL}, 40.0 \mathrm{mmol})$ and $\mathrm{Zn}$ dust $(1.30 \mathrm{~g}, 20.0 \mathrm{mmol})$ and purification by flash column chromatography $\left(\mathrm{CH}_{2} \mathrm{Cl}_{2} / \mathrm{MeOH} 10: 1\right)$ gave diamine 21 (452 mg, $85 \%)$ as a brown oil; $R_{\mathrm{f}} 0.50\left(\mathrm{CH}_{2} \mathrm{Cl}_{2} / \mathrm{MeOH} 10: 1\right)$; IR $v_{\max }$ (thin film): 3374 (br, N-H), 2955 (w, C-H), 1618 (w), 1508 (s, $\mathrm{C}=\mathrm{C}), 1465$ (m), 1441 (w), 1385 (w), 1366 (w), 1292 (w), 1238 (s), 1179 (w), 1154 (w), 1038 (m), 816 (m), 752 (s) cm ${ }^{-1} ;{ }^{1} \mathrm{H}$ NMR (600 MHz) $\delta 0.90\left(\mathrm{~d}, J=6.5,3 \mathrm{H}, \mathrm{CH}_{3}\right), 0.92(\mathrm{~d}, J=6.7$, $\left.3 \mathrm{H}, \mathrm{CH}_{3}\right), 0.99$ (d, $\left.J=6.6,6 \mathrm{H}, \mathrm{CH}_{3}\right), 1.21\left(\mathrm{~m}, 1 \mathrm{H}, \mathrm{CH}_{2}\right), 1.35$ $\left(\mathrm{m}, 1 \mathrm{H}, \mathrm{CH}_{2}\right), 1.59\left(\mathrm{~m}, 1 \mathrm{H}, \mathrm{CH}\left(\mathrm{CH}_{3}\right)_{2}\right), 1.80(\mathrm{~m}, 1 \mathrm{H}$, $\left.\mathrm{C} H\left(\mathrm{CH}_{3}\right)_{2}\right), 2.53\left(\mathrm{dd}, J=9.1,2.7,1 \mathrm{H}, \mathrm{C} H \mathrm{NH}_{2}\right), 3.52(\mathrm{~m}, 1 \mathrm{H}$, $\mathrm{C} H \mathrm{NH}$ ), 3.73 (s, 3H, $\mathrm{OCH}_{3}$ ), 6.57 (app. d, $J=8.9,2 \mathrm{H}, \mathrm{Ar} H$ ), 6.76 (app. d, $J=8.9,2 \mathrm{H}, \mathrm{ArH}) ;{ }^{13} \mathrm{C} \mathrm{NMR}(150 \mathrm{MHz}) \delta 19.6$ $\left(\mathrm{CH}_{3}\right), 20.6\left(\mathrm{CH}_{3}\right), 21.7\left(\mathrm{CH}_{3}\right), 24.1\left(\mathrm{CH}_{3}\right), 24.7\left(\mathrm{CH}\left(\mathrm{CH}_{3}\right)_{2}\right)$, $31.4\left(\mathrm{CH}\left(\mathrm{CH}_{3}\right)_{2}\right), 37.2\left(\mathrm{CH}_{2}\right), 52.9(\mathrm{CHNH}), 55.8\left(\mathrm{OCH}_{3}\right)$, $59.1\left(\mathrm{CHNH}_{2}\right), 114.4$ ( $\mathrm{CH}$ arom. $), 115.0(\mathrm{CH}$ arom. $), 142.3(\mathrm{Cq}$ arom.), 151.6 (Cq arom.); $\mathrm{MS}\left(\mathrm{EI}^{+}\right) \mathrm{m} / z: 264\left(\mathrm{M}^{+}, 5 \%\right), 192$ $\left(\mathrm{M}^{+}-\left(\mathrm{CH}_{3}\right)_{2} \mathrm{CHCHNH}_{2}, 100 \%\right)$; HRMS $\mathrm{m} / \mathrm{z}$ : calcd for $\mathrm{C}_{16} \mathrm{H}_{28} \mathrm{~N}_{2} \mathrm{O}$, 264.2196; found, 264.2201.

$\left(5 S^{*}, 6 R^{*}, Z\right)$-5-Isobutyl-6-isopropyl-4-(4-methoxyphenyl)-3(2-methylpropylidene)piperazin-2-one (23): To a solution of diamine 21 (61 $\mathrm{mg}, 0.23 \mathrm{mmol})$ in THF $(5 \mathrm{~mL})$ was added $N$-(3-dimethylaminopropyl)- $N$ '-ethylcarbodiimide hydrochloride (EDC, $67 \mathrm{mg}, 0.35 \mathrm{mmol}$ ) and 1-hydroxybenzotriazole hydrate $(47 \mathrm{mg}, 0.35 \mathrm{mmol})$ followed by a solution of keto acid 11 (30 mg, $0.23 \mathrm{mmol})$ in $\mathrm{CH}_{2} \mathrm{Cl}_{2}(5 \mathrm{~mL})$ at rt. The 
mixture was stirred for $24 \mathrm{~h}$, then diluted with $\mathrm{CH}_{2} \mathrm{Cl}_{2}(20 \mathrm{~mL})$ and washed with brine $(10 \mathrm{~mL})$. The combined organic phases were dried $\left(\mathrm{MgSO}_{4}\right)$, evaporated in vacuo and purified by flash column chromatography (petrol ether/EtOAc 7:3) to give piperazinone $23\left(57 \mathrm{mg}, 69 \%\right.$ ) as a brown solid; $\mathrm{mp} 152-153{ }^{\circ} \mathrm{C} ; R_{\mathrm{f}}$ 0.30 (petrol ether/EtOAc 7:3); IR $v_{\max }$ (thin film): 3209 (br, N-H), 2956 (w, C-H), 1671 (s, C=C), 1622 (s), 1499 (s), 1464 (m), 1442 (m), 1409 (m), 1384 (m), 1366 (m), 1331 (m), 1283 (m), $1241(\mathrm{~s}), 1180(\mathrm{~m}), 1153(\mathrm{~m}), 1037(\mathrm{~m}), 826(\mathrm{~s}), 767(\mathrm{~m})$ $\mathrm{cm}^{-1} ;{ }^{1} \mathrm{H}$ NMR $(600 \mathrm{MHz}) \delta 0.68\left(\mathrm{~d}, J=6.6,3 \mathrm{H}, \mathrm{CH}_{3}\right), 0.79$ $\left(\mathrm{d}, J=6.6,3 \mathrm{H}, \mathrm{CH}_{3}\right), 0.88\left(\mathrm{~d}, J=6.6,3 \mathrm{H}, \mathrm{CH}_{3}\right), 0.93(\mathrm{~d}, J=$ $\left.6.5,3 \mathrm{H}, \mathrm{CH}_{3}\right), 0.95\left(\mathrm{~d}, J=6.7,3 \mathrm{H}, \mathrm{CH}_{3}\right), 1.07(\mathrm{~d}, J=6.5,3 \mathrm{H}$, $\left.\mathrm{CH}_{3}\right), 1.07\left(\mathrm{~m}, 1 \mathrm{H}, \mathrm{CH}_{2}\right), 1.55\left(\mathrm{~m}, 1 \mathrm{H}, \mathrm{CH}_{2}\right), 1.55(\mathrm{~m}, 1 \mathrm{H}$, $\left.\mathrm{CH}\left(\mathrm{CH}_{3}\right)_{2}\right), 1.94\left(\mathrm{~m}, 1 \mathrm{H}, \mathrm{CH}\left(\mathrm{CH}_{3}\right)_{2}\right), 2.43\left(\mathrm{~m}, 1 \mathrm{H}, \mathrm{CH}\left(\mathrm{CH}_{3}\right)_{2}\right)$, $3.18(\mathrm{dd}, J=10.3,3.6,1 \mathrm{H}, \mathrm{CHNH}), 3.53$ (br. d, $J=12.2,1 \mathrm{H}$, $\mathrm{NCHCH}_{2}$ ), 3.76 (s, 3H, OCH3), 5.89 (br. s, 1H, NH), 6.48 (d, $J$ $=10.6,1 \mathrm{H},=\mathrm{CH}$ ), 6.78 (app. d, $J=8.8,2 \mathrm{H}, \operatorname{Ar} H$ ), 6.89 (app. d, $J=8.8,2 \mathrm{H}, \mathrm{Ar} H) ;{ }^{13} \mathrm{C} \mathrm{NMR}(150 \mathrm{MHz}) \delta 17.9\left(\mathrm{CH}_{3}\right), 19.4$ $\left(\mathrm{CH}_{3}\right), 20.3\left(\mathrm{CH}_{3}\right), 21.5\left(\mathrm{CH}_{3}\right), 22.2\left(\mathrm{CH}_{3}\right), 23.7\left(\mathrm{CH}_{3}\right), 24.1$ $\left(\mathrm{CH}\left(\mathrm{CH}_{3}\right)_{2}\right), 26.4\left(\mathrm{CH}\left(\mathrm{CH}_{3}\right)_{2}\right), 29.0\left(\mathrm{CH}\left(\mathrm{CH}_{3}\right)_{2}\right), 34.4\left(\mathrm{CH}_{2}\right)$, $55.4\left(\mathrm{OCH}_{3}\right), 58.0(\mathrm{CHNH}), 58.8\left(\mathrm{NCHCH}_{2}\right), 114.3(\mathrm{CH}$ arom.), 122.0 ( $\mathrm{CH}$ arom.), $129.7(\mathrm{CH}=\mathrm{Cq}), 140.0(\mathrm{Cq}=\mathrm{CH})$, 143.4 (Cq arom.), 154.3 (Cq arom.), $164.3(C=\mathrm{O})$; $\mathrm{MS}_{\left(\mathrm{EI}^{+}\right)} \mathrm{m} /$ $z: 359\left(\mathrm{MH}^{+}, 100 \%\right), 343$ (12\%), 192 (13\%); HRMS m/z: calcd for $\mathrm{C}_{22} \mathrm{H}_{35} \mathrm{~N}_{2} \mathrm{O}_{2}, 359.2693$; found, 359.2678.

$\left(3 R^{*}, 5 S^{*}, 6 R^{*}\right)-3,5$-diisobutyl-6-isopropyl-4-(4-methoxyphenyl)piperazin-2-one (25): To a solution of piperazinone 23 (170 mg, $0.470 \mathrm{mmol})$ in $\mathrm{MeOH}(10 \mathrm{~mL})$ was added palladium on carbon $(50 \mathrm{mg}, 10 \%$ by weight, $0.047 \mathrm{mmol})$ and the mixture was flushed with hydrogen, then stirred under a hydrogen atmosphere (balloon) at rt. After the piperazinone starting material was consumed (TLC, $4 \mathrm{~h}$ ) the mixture was filtered through celite ${ }^{\circledR}$, washed with $\mathrm{CH}_{2} \mathrm{Cl}_{2}(20 \mathrm{~mL})$ and evaporated in vacuo to give crude piperazinone that was purified by flash column chromatography (petrol ether/ $\mathrm{Me}_{2} \mathrm{CO} 4: 1$ ) to give piperazinone 25 (170 mg, 99\%) as a colourless oil; $R_{\mathrm{f}}$ 0.50 (petrol ether $/ \mathrm{Me}_{2} \mathrm{CO} 4: 1$ ); IR $v_{\max }$ (thin film): 3207 (br, $\mathrm{N}-\mathrm{H}), 2954$ (m, C-H), 1658 (s, C=O), 1505 (C=C), 1465 (m), 1367 (m), 1242 (s), 1180 (m), 1039 (m), 827 (m), 788 (m), 733 (m) $\mathrm{cm}^{-1} ;{ }^{1} \mathrm{H}$ NMR $(600 \mathrm{MHz}) \delta 0.70\left(\mathrm{~d}, J=6.5,3 \mathrm{H}, \mathrm{CH}_{3}\right)$, $0.77\left(\mathrm{~d}, J=6.7,3 \mathrm{H}, \mathrm{CH}_{3}\right), 0.90\left(\mathrm{~d}, J=6.7,3 \mathrm{H}, \mathrm{CH}_{3}\right), 0.97$ (d, $J$ $\left.=6.5,3 \mathrm{H}, \mathrm{CH}_{3}\right), 0.98\left(\mathrm{~d}, J=6.8,3 \mathrm{H}, \mathrm{CH}_{3}\right), 1.03(\mathrm{~d}, J=6.5$, $\left.3 \mathrm{H}, \mathrm{CH}_{3}\right), 1.03\left(\mathrm{~m}, 1 \mathrm{H}, \mathrm{CHCHCH}_{2}\right), 1.55\left(\mathrm{~m}, 1 \mathrm{H}, \mathrm{CHCHCH}_{2}\right)$, $1.55\left(\mathrm{~m}, 1 \mathrm{H}, \mathrm{O}=\mathrm{CCHCH}_{2}\right), 1.55\left(\mathrm{~m}, 1 \mathrm{H}, \mathrm{CHCH}\left(\mathrm{CH}_{3}\right)_{2}\right), 1.84$ $\left(\mathrm{m}, 1 \mathrm{H}, \mathrm{O}=\mathrm{CCHCH}_{2}\right), 1.92\left(\mathrm{~m}, 1 \mathrm{H}, \mathrm{O}=\mathrm{CCHCH}_{2} \mathrm{CH}\left(\mathrm{CH}_{3}\right)_{2}\right)$, $2.06\left(\mathrm{~m}, 1 \mathrm{H}, \mathrm{CHCHCH}_{2} \mathrm{CH}\left(\mathrm{CH}_{3}\right)_{2}\right), 3.18(\mathrm{dd}, J=10.0,3.5$, $1 \mathrm{H}, \mathrm{NHCH}), 3.37(\mathrm{dt}, J=12.4,3.1,1 \mathrm{H}, \mathrm{NHCHC} H \mathrm{~N}), 3.77$ (s, $3 \mathrm{H}, \mathrm{OCH}_{3}$ ), 4.09 (dd, $\left.J=9.9,4.5,1 \mathrm{H}, \mathrm{O}=\mathrm{CCH}\right), 6.02$ (br. s, $1 \mathrm{H}, \mathrm{N} H), 6.80$ (app. d, $J=8.9,2 \mathrm{H}, \operatorname{Ar} H$ ), 6.91 (app. d, $J=8.9$, $2 \mathrm{H}, \mathrm{Ar} H) ;{ }^{13} \mathrm{C}$ NMR $(150 \mathrm{MHz}) \delta 18.0\left(\mathrm{CH}_{3}\right), 19.5\left(\mathrm{CH}_{3}\right), 21.5$
$\left(\mathrm{CH}_{3}\right), 21.6\left(\mathrm{CH}_{3}\right), 23.3\left(\mathrm{CH}_{3}\right), 23.4\left(\mathrm{CHCHCH}_{2} \mathrm{CH}\left(\mathrm{CH}_{3}\right)_{2}\right)$, $23.7\left(\mathrm{CH}_{3}\right), 24.7\left(\mathrm{O}=\mathrm{CCHCH}_{2} \mathrm{CH}\left(\mathrm{CH}_{3}\right)_{2}\right), 29.2$ $\left(\mathrm{NHCHCH}\left(\mathrm{CH}_{3}\right)_{2}\right), 34.7\left(\mathrm{CH}_{2} \mathrm{CHCH}\right), 44.0\left(\mathrm{CH}_{2} \mathrm{CHC}=\mathrm{O}\right)$, $55.4\left(\mathrm{OCH}_{3}\right), 57.2(\mathrm{NCHC}=\mathrm{O}), 58.4(\mathrm{NCHCH}), 58.5(\mathrm{NHCH})$, 114.5 ( $\mathrm{CH}$ arom.), 122.6 ( $\mathrm{CH}$ arom.), 146.2 ( $\mathrm{CH}$ arom.), 154.3 ( $\mathrm{CH}$ arom.), $173.9(C=\mathrm{O})$; $\mathrm{MS}\left(\mathrm{EI}^{+}\right) \mathrm{m} / z: 360\left(\mathrm{M}^{+}, 15 \%\right), 303$ (18\%), 192 (100\%); HRMS $m / z$ : calcd for $\mathrm{C}_{22} \mathrm{H}_{36} \mathrm{~N}_{2} \mathrm{O}_{2}$, 360.2771 ; found, 360.2774 .

\section{$\left(3 R^{*}, 5 S^{*}, 6 R^{*}\right)$-3,5-diisobutyl-6-isopropylpiperazin-2-one}

(2): To a solution of piperazinone $25(320 \mathrm{mg}, 0.880 \mathrm{mmol})$ in $\mathrm{MeCN}(10 \mathrm{~mL})$ at $0{ }^{\circ} \mathrm{C}$ was added a solution of CAN $(2.08 \mathrm{~g}$, $3.52 \mathrm{mmol})$ in $\mathrm{H}_{2} \mathrm{O}(10 \mathrm{~mL})$ dropwise over $3 \mathrm{~min}$. The solution turned from pale yellow to dark orange. The mixture was stirred at $0{ }^{\circ} \mathrm{C}$ for $2 \mathrm{~h}$, over which time the solution became light orange. Water $(30 \mathrm{~mL})$ was then added and the mixture extracted with EtOAc $(3 \times 20 \mathrm{~mL})$, washed with saturated aqueous $\mathrm{NaHCO}_{3}(40 \mathrm{~mL})$, dried $\left(\mathrm{MgSO}_{4}\right)$ and evaporated in vacuo to give crude piperazinone that was purified by flash column chromatography (petrol ether $/ \mathrm{Me}_{2} \mathrm{CO} 3: 2$ ) to give piperazinone $2(91 \mathrm{mg}, 41 \%)$ as a brown oil; $R_{\mathrm{f}} 0.53$ (petrol ether $/ \mathrm{Me}_{2} \mathrm{CO} 3: 2$ ); IR $v_{\max }$ (thin film): 3209 (w, N-H), 2955 (C-H), 1658 (s, C=O), 1467 (m), 1367 (m), 1165 (w), 918 (w), $722(\mathrm{w}) \mathrm{cm}^{-1} ;{ }^{1} \mathrm{H}$ NMR $\left(600 \mathrm{MHz}, \mathrm{CDCl}_{3}\right) \delta 0.89(\mathrm{~d}, J=6.6$, $\left.3 \mathrm{H}, \mathrm{C}-3 \mathrm{CH}_{2} \mathrm{CHCH}_{3}\right), 0.90\left(\mathrm{~d}, J=6.2,3 \mathrm{H}, \mathrm{C}-5 \mathrm{CH}_{2} \mathrm{CHCH}_{3}\right)$, $0.91\left(\mathrm{~d}, J=5.6,3 \mathrm{H}, \mathrm{C}-6 \mathrm{CHCH}_{3}\right), 0.93(\mathrm{~d}, J=6.9,3 \mathrm{H}$, $\mathrm{C}-3 \mathrm{CH}_{2} \mathrm{CHCH}_{3}$ ), 0.94 (d, $\left.J=6.8,3 \mathrm{H}, \mathrm{C}-5 \mathrm{CH}_{2} \mathrm{CHCH}_{3}\right), 0.98$ $\left(\mathrm{d}, J=6.7,3 \mathrm{H}, \mathrm{C}-6 \mathrm{CHCH}_{3}\right), 1.30(\mathrm{ddd}, J=13.9,7.1,6.5,1 \mathrm{H}$, C-5 $\mathrm{CH}_{2}$ ), 1.33 (ddd, $J=13.9,8.2,5.6,1 \mathrm{H}, \mathrm{C}-5 \mathrm{CH}_{2}$ ), 1.40 (ddd, $J=14.2,10.0,4.1,1 \mathrm{H}, \mathrm{C}-3 \mathrm{CH}_{2}$ ), 1.65 (nonet, $J=6.7,1 \mathrm{H}$, $\left.\mathrm{C}-5 \mathrm{CH}_{2} \mathrm{CH}\right), 1.74\left(\mathrm{~m}, 1 \mathrm{H}, \mathrm{C}-3 \mathrm{CH}_{2} \mathrm{CH}\right), 1.88(\mathrm{ddd}, J=13.7$, $10.3,3.3,1 \mathrm{H}, \mathrm{C}-3 \mathrm{CH}_{2}$ ), 1.91 (hepd, $J=6.8,2.5,1 \mathrm{H}, \mathrm{C}-6 \mathrm{CH}$ ), $3.06(\mathrm{dd}, J=6.7,3.6,1 \mathrm{H}, \mathrm{C}-6 H), 3.15(\mathrm{dt}, J=7.8,5.3,1 \mathrm{H}$, C-5H), 3.40 (dd, $J=10.2,3.4,1 \mathrm{H}, \mathrm{C}-3 H), 6.22$ (brs, $1 \mathrm{H}, \mathrm{N}^{1} H$ ), $\mathrm{N}^{4} H$ peak is missing; ${ }^{13} \mathrm{C} \mathrm{NMR}\left(125 \mathrm{MHz}, \mathrm{CDCl}_{3}\right) \delta 17.7$ $\left(\mathrm{C}-6 \mathrm{CHCH}_{3}\right), 21.0\left(\mathrm{C}-3 \mathrm{CH}_{2} \mathrm{CHCH}_{3}\right), 21.5\left(\mathrm{C}-5 \mathrm{CH}_{2} \mathrm{CHCH}_{3}\right)$, $22.4\left(\mathrm{C}-6 \mathrm{CHCH}_{3}\right), 23.1$ and $23.7\left(\mathrm{C}-3 \mathrm{CH}_{2} \mathrm{CHCH}_{3} /\right.$ $\left.\mathrm{C}-5 \mathrm{CH}_{2} \mathrm{CHCH}_{3}\right), 24.4\left(\mathrm{C}-3 \mathrm{CH}_{2} \mathrm{CH}\right), 24.8\left(\mathrm{C}-5 \mathrm{CH}_{2} \mathrm{CH}\right), 27.9$ $(\mathrm{C}-6 \mathrm{CH}), 40.5\left(\mathrm{C}-5 \mathrm{CH}_{2}\right), 41.1\left(\mathrm{C}-3 \mathrm{CH}_{2}\right), 53.3(\mathrm{C}-5), 57.0$ (C-3), $59.4(\mathrm{C}-6), 174.2(\mathrm{C}=\mathrm{O})$; $\mathrm{MS}\left(\mathrm{EI}^{+}\right) \mathrm{m} / z: 254\left(\mathrm{M}^{+}, 30 \%\right)$, 197 (22\%), 169 (43\%), 154 (31\%); HRMS $m / z$ : calcd for $\mathrm{C}_{15} \mathrm{H}_{30} \mathrm{~N}_{2} \mathrm{O}$, 254.2353; found, 254.2355.

${ }^{1} \mathrm{H}$ NMR (600 MHz, DMSO- $\left.d_{6}\right) \delta$ 0.83-0.89 (m, 18H, 6xMe), $1.25\left(\mathrm{~m}, 3 \mathrm{H}, \mathrm{C}-3 \mathrm{CH}_{2}+2 \mathrm{xC}-5 \mathrm{CH}_{2}\right), 1.65\left(\mathrm{~m}, 2 \mathrm{H}, \mathrm{C}-3 \mathrm{CH}_{2} \mathrm{CH}+\right.$ $\left.\mathrm{C}-5 \mathrm{CH}_{2} \mathrm{CH}\right), 1.79\left(\mathrm{~m}, 2 \mathrm{H}, \mathrm{C}-3 \mathrm{CH}_{2}+\mathrm{C}-6 \mathrm{CH}\right), 2.90$ (brd, $J=$ $3.2,1 \mathrm{H}, \mathrm{C}-6 H), 3.01(\mathrm{td}, J=7.1,3.7,1 \mathrm{H}, \mathrm{C}-5 H), 3.21(\mathrm{dd}, J=$ 9.3, 3.2, 1H, C-3H), 7.63 (brs, $1 \mathrm{H}, \mathrm{NH}$ ). Assignments based on above; ${ }^{13} \mathrm{C}$ NMR $\left(125 \mathrm{MHz}, \mathrm{DMSO}-d_{6}\right) \delta 18.4,21.5,22.0$, 22.8, 23.0, 23.8, 23.9, 24.2, 27.4, 40.2, 41.5, 53.2, 56.4, 58.1, 172.5 . 
In a clean vial $\mathrm{HCl}$ in dioxane ( $\sim$ equiv) was added to a small sample of $\mathbf{2}$ in $\mathrm{MeCN}$, the mixture layered with $\mathrm{Et}_{2} \mathrm{O}$ and then left in the fridge until colourless blades of $2 \cdot \mathrm{HCl}$ had formed at the interface. mp $121-123{ }^{\circ} \mathrm{C} .{ }^{1} \mathrm{H}$ NMR $\left(600 \mathrm{MHz}, \mathrm{D}_{2} \mathrm{O}\right) \delta$ 0.89-0.99 (6xMe), 1.52-1.68 (m, 4H), 1.72-1.79 (m, 1H), $1.94-1.99(\mathrm{~m}, 2 \mathrm{H}), 3.50(\mathrm{dd}, J=6.2,4.3,1 \mathrm{H}, \mathrm{C}-6 H), 3.79(\mathrm{~m}$, $1 \mathrm{H}, \mathrm{C}-5 H), 4.01(\mathrm{dd}, J=8.3,5.3,1 \mathrm{H}, \mathrm{C}-3 H) ;{ }^{13} \mathrm{C}$ NMR $(150$ $\left.\mathrm{MHz}, \mathrm{D}_{2} \mathrm{O}\right) \delta 18.8,20.6,21.3,22.0,22.5,24.2,24.7,25.7,27.4$, $36.3,38.8,53.9,54.9,56.3,169.9(\mathrm{C}=\mathrm{O})$.

\section{Supporting Information}

\section{Supporting Information File 1}

Further experimental and characterisation data.

[http://www.beilstein-journals.org/bjoc/content/ supplementary/1860-5397-9-200-S1.pdf]

\section{Acknowledgements}

We thank the Cyprus State Scholarship Foundation for funding, Dr. L. Haigh and Mr. J. Hill for providing mass spectra and Ms. J. Maxwell for microanalytical data.

\section{References}

1. Noble, A.; Anderson, J. C. Chem. Rev. 2013, 113, 2887. doi:10.1021/cr300272t

See for a comprehensive review of the nitro-Mannich reaction.

2. Ballini, R.; Petrini, M. Tetrahedron 2004, 60, 1017. doi:10.1016/j.tet.2003.11.016

3. Palomo, C.; Oiarbide, M.; Halder, R.; Laso, A.; López, R. Angew. Chem., Int. Ed. 2006, 45, 117. doi:10.1002/anie.200502674

4. Bursavich, M. G.; Rich, D. H. J. Med. Chem. 2002, 45, 541. doi:10.1021/jm010425b

5. Shen, B.; Johnston, J. N. Org. Lett. 2008, 10, 4397. doi:10.1021/ol801797h

6. Jakubec, P.; Cockfield, D. M.; Dixon, D. J. J. Am. Chem. Soc. 2009, 131, 16632. doi:10.1021/ja908399s

7. Jakubec, P.; Kyle, A. F.; Calleja, J.; Dixon, D. J. Tetrahedron Lett. 2011, 52, 6094. doi:10.1016/j.tetlet.2011.09.016

8. Kyle, A. F.; Jakubec, P.; Cockfield, D. M.; Cleator, E.; Skidmore, J.; Dixon, D. J. Chem. Commun. 2011, 47, 10037. doi:10.1039/c1cc13665h

9. Kumaraswamy, G.; Pitchaiah, A. Helv. Chim. Acta 2011, 94, 1543. doi:10.1002/hlca.201100013

10. Jakubec, P.; Hawkins, A.; Felzmann, W.; Dixon, D. J. J. Am. Chem. Soc. 2012, 134, 17482. doi:10.1021/ja308826x

11. Tsuritani, N.; Yamada, K.-I.; Yoshikawa, N.; Shibasaki, M. Chem. Lett. 2002, 31, 276. doi:10.1246/cl.2002.276

12. Xu, X.; Furukawa, T.; Okino, T.; Miyabe, H.; Takemoto, Y. Chem.-Eur. J. 2006, 12, 466. doi:10.1002/chem.200500735

13. Anderson, J. C.; Chapman, H. A. Org. Biomol. Chem. 2007, 5, 2413-2422. doi:10.1039/b705081j

14. Hynes, P. S.; Stupple, P. A.; Dixon, D. J. Org. Lett. 2008, 10, 1389. doi:10.1021/ol800108u
15. Handa, S.; Gnanadesikan, V.; Matsunaga, S.; Shibasaki, M. J. J. Am. Chem. Soc. 2010, 132, 4925. doi:10.1021/ja100514y 16. Weng, J.; Li, Y.-B.; Wang, R.-B.; Li, F.-Q.; Liu, C.; Chan, A. S. C.; Lu, G. J. Org. Chem. 2010, 75, 3125. doi:10.1021/jo100187m

17. Kumaraswamy, G.; Pitchaiah, A. Tetrahedron 2011, 67, 2536. doi:10.1016/j.tet.2011.02.031

18. Davis, T. A.; Johnston, J. N. Chem. Sci. 2011, 2, 1076. doi:10.1039/c1sc00061f

19. Xie, H.; Zhang, Y.; Zhang, S.; Chen, X.; Wang, W. Angew. Chem., Int. Ed. 2011, 50, 11773. doi:10.1002/anie.201105970

20. Jensen, K. L.; Poulsen, P. H.; Donslund, B. S.; Morana, F.; Jørgensen, K. A. Org. Lett. 2012, 14, 1516. doi:10.1021/ol3002514

21. Anderson, J. C.; Horsfall, L. R.; Kalogirou, A. S.; Mills, M. R.; Stepney, G. J.; Tizzard, G. J. J. Org. Chem. 2012, 77, 6186-6198. doi:10.1021/jo301000r

22. Davis, T. A.; Danneman, M. W.; Johnston, J. N. Chem. Commun. 2012, 48, 5578. doi:10.1039/c2cc32225k

23. Anderson, J. C.; Noble, A.; Tocher, D. A. J. Org. Chem. 2012, 77, 6703. doi:10.1021/jo3010827

24. Anderson, J. C.; Noble, A.; Ribelles Torres, P. Tetrahedron Lett. 2012, 53, 5707. doi:10.1016/j.tetlet.2012.08.062

25. Marquéz-López, E.; Merino, P.; Tejero, T.; Herrera, R. P. Eur. J. Org. Chem. 2009, 2401. doi:10.1002/ejoc.200801097 See for a review specific to asymmetric methods.

26. Yamada, K.-I.; Moll, G.; Shibasaki, M. Synlett 2001, 980 doi:10.1055/s-2001-14639

27. Knudsen, K. R.; Risgaard, T.; Nishiwaki, N.; Gothelf, K. V.; Jørgensen, K. A. J. Am. Chem. Soc. 2001, 123, 5843. doi:10.1021/ja010588p

28. Anderson, J. C.; Howell, G. P.; Lawrence, R. M.; Wilson, C. S. J. Org. Chem. 2005, 70, 5665. doi:10.1021/jo050762i

29. Trost, B. M.; Lupton, D. W. Org. Lett. 2007, 9, 2023. doi:10.1021/ol070618e

30. Chen, Z.; Morimoto, H.; Matsunaga, S.; Shibasaki, M. J. Am. Chem. Soc. 2008, 130, 2170. doi:10.1021/ja710398q

31. Yamada, K.-I.; Harwood, S. J.; Gröger, H.; Shibasaki, M. Angew. Chem., Int. Ed. 1999, 38, 3504. doi:10.1002/(SICI)1521-3773(19991203)38:23<3504::AID-ANIE3504>3 .0.CO;2-E

32. Handa, S.; Gnanadesikan, V.; Matsunaga, S.; Shibasaki, M. J. Am. Chem. Soc. 2007, 129, 4900. doi:10.1021/ja0701560

33. Okino, T.; Nakamura, S.; Furukawa, T.; Takemoto, Y. Org. Lett. 2004, 6, 625. doi:10.1021/ol0364531

34. Nugent, B. M.; Yoder, R. A.; Johnston, J. N. J. Am. Chem. Soc. 2004, 126, 3418. doi:10.1021/ja031906i

35. Yoon, T. P.; Jacobsen, E. N. Angew. Chem., Int. Ed. 2005, 44, 466. doi:10.1002/anie.200461814

36. Fini, F.; Sgarzani, V.; Petterson, D.; Herrera, R. P.; Bernardi, L.; Ricci, A. Angew. Chem., Int. Ed. 2005, 44, 7975. doi:10.1002/anie.200502646

37. Palomo, C.; Oiarbide, M.; Laso, A.; López, R. J. Am. Chem. Soc. 2005, 127, 17622. doi:10.1021/ja056594t

38. Robak, M. T.; Trincado, M.; Ellman, J. A. J. Am. Chem. Soc. 2007, 129, 15110. doi:10.1021/ja075653v

39. Wang, C.-J.; Dong, X.-Q.; Zhang, Z.-H.; Xue, Z.-Y.; Teng, H.-L. J. Am. Chem. Soc. 2008, 130, 8606. doi:10.1021/ja803538x

40. Adams, H.; Anderson, J. C.; Peace, S.; Pennell, A. M. K. J. Org. Chem. 1998, 63, 9932. doi:10.1021/jo981700d

41. Anderson, J. C.; Peace, S.; Pih, S. Synlett 2000, 850. doi:10.1055/s-2000-6710 
42. Anderson, J. C.; Blake, A. J.; Howell, G. P.; Wilson, C. J. Org. Chem. 2005, 70, 549. doi:10.1021/jo048304h

43. Anderson, J. C.; Stepney, G. J.; Mills, M. R.; Horsfall, L. R.; Blake, A. J.; Lewis, W. J. Org. Chem. 2011, 76, 1961. doi:10.1021/jo102408u

44. Anderson, J. C.; Blake, A. J.; Koovits, P. J.; Stepney, G. J. J. Org. Chem. 2012, 77, 4711. doi:10.1021/j0300535h

45. Anderson, J. C.; Koovits, P. J. Chem. Sci. 2013, 4, 2897. doi:10.1039/c3sc50613d

46. El-Desouky, S.-K.; Ryu, S. Y.; Kim, Y.-K. Tetrahedron Lett. 2007, 48 , 4015. doi:10.1016/j.tetlet.2007.04.032

47. Rambaud, M.; Bakasse, M.; Duguay, G.; Villieras, J. Synthesis 1988, 564. doi:10.1055/s-1988-27642

48. Ramage, R.; Griffiths, G. J.; Shutt, F. E.; Sweeney, J. N. A. J. Chem. Soc., Perkin Trans. 1 1984, 1531. doi:10.1039/P19840001531

49. Fuse, S.; Masui, H.; Tannna, A.; Shimizu, F.; Takahashi, T. ACS Comb. Sci. 2012, 14, 17. doi:10.1021/co200081j

50. Kumaran, G.; Kulkarni, G. H. Synthesis 1995, 1545. doi:10.1055/s-1995-4137

51. Boeykens, M.; De Kimpe, N.; Tehrani, K. A. J. Org. Chem. 1994, 59, 6973. doi:10.1021/jo00102a022

52. Li, L.-S.; Zhou, Y.; Zhao, J.; Dragovich, P. S.; Stankovic, N.; Bertolini, T. M.; Murphy, D. E.; Sun, Z.; Tran, C. V.; Ayida, B. K.; Ruebsam, F.; Webber, S. E. Synthesis 2007, 3301. doi:10.1055/s-2007-990823

53. Murthy, S. N.; Madhav, B.; Nageswar, Y. V. D. Helv. Chim. Acta 2010, 93, 1216. doi:10.1002/hlca.200900358

54. Lambert, A.; Lowe, A. J. Chem. Soc. 1947, 1517. doi:10.1039/jr9470001517

55. Reaction in THF gave a lower stereoselectivity of $d r$ 85:15. The use of $\mathrm{CH}_{2} \mathrm{Cl}_{2}$ as solvent in reductive nitro-Mannich reactions can give enhanced diastereoselectivities. See [24].

56. Kaliogirou, A. S. Ph.D. Thesis, University College London, London, 2013.

57. Grijalvo, S.; Bedia, C.; Triola, G.; Casas, J.; Llebaria, A.; Teixidó, J.; Rabal, O.; Levade, T.; Delgado, A.; Fabriàs, G. Chem. Phys. Lipids 2006, 144, 69. doi:10.1016/j.chemphyslip.2006.07.001

58. The publication [49] states in the discussion that the NMR were recorded in DMSO- $d_{6}$, but is then tabulated in $\mathrm{D}_{2} \mathrm{O}$. The author confirmed the data was recorded in $\mathrm{D}_{2} \mathrm{O}$ and that there were no suitable samples of the material left for re-analysis and the original spectra were unobtainable. Private communication from Prof Young-Kyoon Kim.

59. Crystallographic data (excluding structure factors) for the structure of 2. $\mathrm{HCl}$ has been deposited with the Cambridge Crystallographic Data Centre under supplementary publication numbers CCDC 949530. Copies of the data can be obtained, free of charge, upon request from the CCDC, 12 Union Road, Cambridge CB2 1EZ, UK [fax: +44(0)-1223-336033 or email: deposit@ccdc.cam.ac.uk].

60. Perrin, D. D.; Armarego, W. L. F.; Perrin, D. R. Purification of Laboratory Chemicals, 2nd ed.; Pergamon Press Ltd., 1980.

61. Oiima, I.; Habus, H.; Zhao, M.; Zucco, M.; Park, Y. H.; Sun, C. M.; Brigaud, T. Tetrahedron 1992, 48, 6985. doi:10.1016/S0040-4020(01)91210-4

\section{License and Terms}

This is an Open Access article under the terms of the Creative Commons Attribution License

(http://creativecommons.org/licenses/by/2.0), which permits unrestricted use, distribution, and reproduction in any medium, provided the original work is properly cited.

The license is subject to the Beilstein Journal of Organic Chemistry terms and conditions:

(http://www.beilstein-journals.org/bjoc)

The definitive version of this article is the electronic one which can be found at:

doi:10.3762/bjoc. 9.200 